\title{
Les stocks et la volatilité des prix agricoles. Un modèle de fluctuations endogènes
}

Storage and the Volatility of Agricultural Prices: a Model of Endogenous

Fluctuations

\section{Sophie Mitra et Jean-Marc Boussard}

\section{(2) OpenEdition}

\section{Journals}

Édition électronique

URL : http://journals.openedition.org/economierurale/2925

DOI : 10.4000/economierurale.2925

ISSN : 2105-2581

\section{Éditeur}

Société Française d'Économie Rurale (SFER)

Édition imprimée

Date de publication : 6 janvier 2011

Pagination : Pagination

ISSN : 0013-0559

Référence électronique

Sophie Mitra et Jean-Marc Boussard, «Les stocks et la volatilité des prix agricoles. Un modèle de fluctuations endogènes », Économie rurale [En ligne], 321 | janvier-février 2011, mis en ligne le 06 janvier 2013, consulté le 02 mai 2019. URL : http://journals.openedition.org/economierurale/2925 ; DOI : 10.4000/economierurale.2925 


\section{Les stocks et la volatilité des prix agricoles Un modèle de fluctuations endogènes}

Sophie MITRA - Department of Economics, Fordham University, New York, USA

Jean-Marc BOUSSARD • Institut national de recherche en agronomie (INRA), Paris, France

\section{Introduction}

a récente crise des prix des matières $\checkmark$ premières agricoles mondiales remet à l'ordre du jour la question de la volatilité des prix alimentaires, et des explications à donner à ce phénomène. Il existe un assez large consensus sur le fait que ces fluctuations sont néfastes, en écartant durablement le prix de marché du coût marginal de longue période, et en créant des difficultés au niveau de la réduction de la pauvreté et de la sécurité alimentaire (Ravallion, 1997). Cependant, la question de l'origine de ces fluctuations - et par conséquent des actions qui pourraient être prises pour y remédier - reste pendante.

Sans doute existe-t-il chez les économistes un accord au moins sur un point : dans tous les cas, l'inélasticité de la demande conduit à amplifier de faibles mouvements de l'offre, provoquant ainsi d'énormes variations des prix à partir de causes à première vue ténues. Quant aux mouvements de l'offre, il existe deux grandes écoles sur leurs origines.

\section{Les deux hypothèses sur l'origine des fluctuations}

L'hypothèse la plus communément acceptée est celle des fluctuations d'origine aléatoire ou exogène (Cafiero, Wright, 2006 ; Deaton, Laroque, 1992, 1996) : la production agricole est sujette à de nombreux accidents d'origine météorologique ${ }^{1}$, complètement extérieurs au marché, mais qui perturbent l'offre et la demande. Les producteurs sont supposés neutres vis-à-vis du

1. Ainsi, la sécheresse en Australie a parfois été présentée comme l'un des principaux facteurs de la crise récente, voir par exemple « Drought a Factor in Food Aid Shortage : UN » The Australian 13/09/2009. risque et avoir des anticipations rationnelles, ce qui n'est pas réaliste (Nerlove, Bessler, 2001). L'autre difficulté avec cette hypothèse est que des perturbations aléatoires ne produisent pas les faits stylisés des séries de prix agricoles, à savoir la dissymétrie de la densité de probabilité et l'auto-corrélation d'ordre un.

L'autre hypothèse, au contraire, s'attache à des causes endogènes, liées au fonctionnement même du marché, et conduisant parfois à des phénomènes chaotiques ${ }^{2}$. Par exemple, Ezekiel (1938) met en avant les erreurs d'anticipation des producteurs, qui conduisent à des alternances de sur- et de sous-production. Ici encore, l'hypothèse de Ezekiel à l'état brut conduit à envisager des mouvements de prix périodiques, divergents ou convergents, qui ne correspondent en rien à la réalité.

Il y a une part de vérité dans chacune de ces deux hypothèses. Toutefois, il est essentiel de savoir laquelle des deux explique le mieux les fluctuations observées, car elles ont des implications pratiques très différentes, et même opposées pour les politiques à mettre en œuvre afin de réduire les effets néfastes de ces phénomènes.

\section{L'importance de trancher}

Si l'hypothèse exogène est la bonne, il devrait être possible de remédier aux fluctuations en faisant jouer la loi des grands nombres, à travers des mécanismes d'assurance, que ce soit les assurances traditionnelles, qui mutua-

2. Le mot « chaos » est ici pris dans le sens qu'il a en dynamique mathématique : il s'applique à la solution d'une équation différentielle lorsqu'elle ne converge jamais vers une limite stable après un temps plus ou moins long, sans cependant être périodique. Cf. Aligood et al. (1996). 
lisent les risques entre « chanceux » et « malchanceux » à un instant donné, le stockage (la Loi des grands nombres permet alors une mutualisation des risques dans le temps) ou l'élargissement des marchés (avec mutualisation des risques dans l'espace). De fait, la loi des grands nombres dit que nombreux risques, chacun d'eux « petits » vis-à-vis de l'ensemble, et indépendants en probabilité, s'annulent mutuellement. Ainsi, l'existence d'une sécheresse qui affecterait à la fois l'Australie, l'Europe et les États-Unis semble très peu probable, même si la probabilité d'un tel évènement au niveau de chacun de ces continents n'est pas négligeable ${ }^{3}$. De la même manière, si une mauvaise récolte peut survenir une année, une longue série de mauvaises récoltes est peu crédible. Dans un tel contexte, l'élargissement des marchés semble être une bonne méthode pour réduire les fluctuations. C'est l'une des raisons pour laquelle la « libéralisation » de l'agriculture avait été recommandée par les auteurs qui avaient préparé les négociations de l'Organisation mondiale du commerce (OMC) lors de l'Uruguay round ${ }^{4}$, puis, plus tard, à Doha. De même, dans cette optique, l'existence d'un stock de sécurité interannuel devrait aussi permettre de réguler les prix : c'est bien pourquoi, depuis la nuit des temps ${ }^{5}$, les stocks

\footnotetext{
3. À noter que selon Cobble, Dismuke et Thomas (2007), si le coefficient de variation des rendements est de l'ordre de $25 \%$ pour le maïs ou $39 \%$ pour le coton au niveau de l'exploitation aux USA, il n'est plus que de $15 \%$ pour le maïs ; $24 \%$ pour le coton au niveau des états de l'Union et tombe à 8 et $11 \%$ lorsqu'il est évalué au niveau de l'Union. 4. Ainsi, Bale et Lutz (1979), une référence qui a eu un succès considérable, quoiqu'il ne s'agisse que d'une démonstration d'ailleurs un peu fruste du théorème de la Loi des grands nombres.

5. Que 1'on se rappelle l'histoire des « vaches grasses » et des « vaches maigres » dans la Bible, à la fin du livre de la Genèse, qui sert au Pharaon d'Égypte à justifier une politique de stockage/déstockage de grains de nature à lui assurer l'hégémonie sur tout le Moyen-Orient, à travers une crise de liquidités internationale. $C f$. Genèse, 41-50.
}

publics de sécurité sont le cœur des politiques de sécurité alimentaire.

Au contraire, avec des fluctuations endogènes, de tels remèdes ont peu de chances de résoudre le problème. L'élargissement des marchés conduira seulement à les synchroniser, pas à réduire les fluctuations. De tels remèdes ont peu de chances de résoudre le problème, car la Loi des grands nombres ne fonctionne plus. Sans doute, tous les producteurs n'ont-ils pas les mêmes anticipations, et celles-ci peuvent se trouver plus ou moins dispersées autour d'une moyenne. Mais il y a très peu de chance pour que cette moyenne se situe au niveau idéal de l'anticipation « rationnelle », qui égaliserait le prix moyen avec le coût marginal. S'il y a des raisons de croire que le prix va augmenter, tout le monde va parier en même temps sur cet accroissement, de sorte que même si les prévisions diffèrent d'un opérateur à l'autre, la moyenne de toutes les anticipations sera belle et bien à la hausse, et c'est justement cela qui fera la baisse ! Depuis très longtemps, de nombreux auteurs (Mandelbrot, 1973 ; Orléans, 1989), ont souligné l'importance de ces phénomènes, qui peuvent expliquer pourquoi tous les chocs sont corrélés entre eux, ce qui supprime l'une des conditions de validité de la Loi des grands nombres, et conduit, pour les variables macroéconomiques à des distributions qui n'ont plus rien de gaussien (Zajdenweber, 2000).

Lorsque de tels phénomènes sont en jeu, la simple addition des variables ne suffit plus à en régulariser la somme, parce que chaque variable individuelle dépend en fait du même mécanisme sous jacent, commun à toutes. De ce fait, un procédé de régularisation qui semble naturel, comme l'élargissement des marchés, au lieu d'atténuer les fluctuations, conduira seulement à les synchroniser. Dans ce contexte, il est possible d'envisager que le stockage public ou privé puisse se produire à contretemps, ce qui, au lieu de diminuer l'ampleur de la crise, pourrait conduire plutôt à l'aggraver, point 
sur lequel nous revenons dans le modèle ci-dessous. Inversement, segmenter les marchés et développer des interventions publiques sur les prix et les quantités peut contribuer à les diminuer, à un coût bientôt compensé par les avantages de la stabilisation (Boussard, 2006).

\section{Les travaux passés}

En dépit de l'importance de savoir si les fluctuations sont endogènes ou exogènes, très peu de références sont disponibles sur la question. On a parfois essayé de tester directement le caractère chaotique de certaines séries (par exemple Leuthold, Wei, 1998). Malheureusement, rien n'est plus semblable à une série aléatoire qu'une série chaotique, de sorte que le caractère discriminant de ces tests est assez faible. Une autre approche consiste à construire un modèle explicite du fonctionnement des marchés dans l'hypothèse endogène, et analyser si les séries obtenues sont semblables à des séries « réelles ».

De ce point de vue, dans une étude remarquable et célèbre, Deaton et Laroque (1992, 1996) montrent en effet que, dans le contexte de l'hypothèse exogène, les caractéristiques majeures des séries de prix de matières premières agricoles (en particulier, Skewness ${ }^{6}$ et auto-corrélation d'ordre un) peuvent être obtenues à partir de perturbations aléatoires « $\mathrm{iid}^{7} » \mathrm{de}$

\footnotetext{
6. Skewness : La Skewness est un nombre qui mesure le degré de dissymétrie d'une distribution de probabilité. Souvent employé en français, c'est un anglicisme. Pourtant, même en anglais, le mot ne figure pas dans les dictionnaires comme le Collins ou le Concise Oxford Dictionnary : c'est donc un néologisme forgé par les mathématiciens à partir de skew qui comme adjectif veut dire : penché, tordu ou oblique. C'est pourquoi ici, nous remplacerons skewness par obliquité ( $C f$. dictionnaire Robert). Le Comité de rédaction laisse toute latitude d'interprétation au lecteur mais se doit de respecter le choix de l'auteur [NdR].

7. Chaque aléa est distribué de façon identique au précédent, et indépendant de celui-ci.
}

l'offre (perturbations dont, bien sûr, la densité de probabilité a une allure très différente de celle que l'on peut observer pour les $\left.\operatorname{prix}^{8}\right)$. Il faut pour cela que la série des perturbations de l'offre soit transformée par le comportement des stockeurs privés, qui observent le niveau des stocks existants, et se mettent à stocker lorsque les stocks sont bas, afin de profiter de la hausse des prix que l'on peut attendre de cette situation. Avec le stockage, Deaton et Laroque ont ainsi introduit dans leur modèle une «mémoire » qui pourrait expliquer l'auto-corrélation d'ordre 1 généralement observée dans les séries réelles. De plus, du fait que les stocks ont une grande probabilité de devenir nul un jour ou l'autre, on peut obtenir par là des séries de prix caractérisées par une grande dissymétrie. Les prix sont bas la plupart du temps, mais quand, à intervalles assez éloignés les uns des autres, les stocks sont vides, ils deviennent très élevés.

L'expérience de Deaton et Laroque permettrait par conséquent de conclure que la volatilité des prix agricoles est causée par des perturbations aléatoires, puisqu'il est possible de reproduire les caractéristiques observées des séries de prix à partir de cette hypothèse. Ici, nous allons montrer que cette expérience ne tranche pas la question, puisqu'il est aussi possible de reproduire ces caractéristiques à partir de l'hypothèse endogène. Nous commencerons par un bref survol des modèles « chaotiques » de fonctionnement des marchés agricoles. Puis nous exposerons notre propre modèle, et en détaillerons les résultats. Enfin, nous tirerons les leçons de l'expérience.

8. Deaton et Laroque ne sont pas complètement satisfaits de leur expérience, pas plus que nous ne le sommes de la notre. En particulier, leur coefficient d'auto-corellation d'ordre 1 n'est pas satisfaisant. Mais ils considèrent au moins qu'ils se sont rapprochés de la vérité, ce qui est notre cas aussi, comme on va le voir. 


\section{Les modèles chaotiques du fonctionnement des marchés agricoles}

Depuis le début des années 1990, plusieurs modèles de la dynamique des prix agricoles ont identifié des sources possibles pour un régime chaotique des prix. Tous impliquent : 1. L'instabilité locale du système d'offre et de demande au voisinage du point d'équilibre du marché, à cause de la rigidité de la demande. Le point d'équilibre est alors « répulsif », conduisant le système à l'en éloigner encore plus dès lors qu'il n'est pas exactement dessus (comme c'est le cas d'une bille à la pointe d'un crayon). Chavas et Holt (1993) ont en effet montré que la rigidité de la demande est un facteur de chaos au sein des marchés agricoles. Ils utilisent un modèle du marché laitier americain. Ils trouvent que plus la demande est inélastique, plus l'exposant de Lyapunov ${ }^{9}$ augmente, en d'autres termes, plus la dynamique du modèle devient chaotique. 2. Un autre mécanisme qui tend à ramener le système en direction du point d'équilibre lorsqu'il s'en éloigne trop (comme la boule d'un bilboquet, qui est rattachée à la poignée par une ficelle). Cet autre mécanisme a pris plusieurs formes dans cette littérature. Chavas et Holt (1993) utilisent des courbes d'offre et de demande nonlinéaires. Finkenstadt et Kuhbier (1992) présentent un modèle de cobweb qui combine une demande non linéaire et des anticipations adaptatives. Boussard (1996) conserve une demande linéaire mais utilise des espérances constantes ou naïves, et suppose que le producteur a de l'aversion pour le risque.
Ces modèles ont pu démontrer qu'il est possible d'obtenir des fluctuations purement endogènes lorsqu'on abandonne les hypothèses de linéarité, de rationalité et de neutralité vis-à-vis du risque qui sont typiques des modèles traditionnels de fluctuations exogènes. Cependant, ces travaux sur les marchés de matières premières agricoles ont l'inconvénient de ne pas étudier les caractéristiques empiriques des séries obtenues à partir de modèles chaotiques pour savoir s'ils permettent de reproduire les « faits stylisés » relatifs aux prix agricoles, en particulier leur obliquité positive et leur auto-corrélation de rang 1. En réalité, si l'on consulte la littérature générale sur le chaos, les modèles, la plupart du temps, conduisent à des séries de prix simulés qui exhibent une obliquité négative, et un coefficient d'auto-corrélation du premier ordre assez faible (Brock, Dindo, Hommes, 2007 ; Hommes, 1998 ; Hommes, van Eekelen, 1996), conduisant à considérer qu'ils ne correspondent pas à la réalité observée. Mais dans le cas du modèle de Deaton et Laroque, on se trouvait en fait dans la même situation, avec au départ une série de quantités offertes dépourvue d'auto-corrélation, et distribuée de façon parfaitement symétrique. C'est seulement le stockage qui transformait cette perturbation iid en une fonction de répartition dissymétrique, et dotée d'autocorrélation. Ne pourrait-on faire de même avec une série chaotique, comme celles dont il vient d'être question pour les prix agricoles ? C'est ce qui est tenté ici, et qui fait l'originalité de ce travail. 


\section{Pour permettre une lecture plus aisée des simulations numériques et des équations du modèle,} cette section est présentée sur pleine page (NDLR).

\section{Un nouveau modèle intégrant le stockage}

On considère un produit agricole qui est récolté annuellement et consommé durant deux saisons. Les décisions de production sont prises en saison 1 (l'automne), les décisions de stockage et de consommation en saison 2 (l'été). Les quantités plantées en automne sont livrées sur le marché en été. Le marché est segmenté entre un marché local, où l'agriculteur vend sa marchandise à un commerçant, et un marché central, où ce dernier la revend au consommateur. Ce scénario est spécialement adapté au cas des pays en voie de développement, mais il peut aussi correspondre à celui des pays développés (Bobenrieth, Bobenrieth et Wright, 2006).

Soient $p_{t, i}^{\mathrm{c}}$ et $q_{t, i}^{\mathrm{c}}$, respectivement, les prix et quantités sur le marché central au cours de la saison $i$ de l'année $t$. La décision de production est prise en automne $(i=1)$, avec un coût marginal linéaire :

$$
p_{t, 1}^{\mathrm{c}}=a q_{t, 1}^{\mathrm{c}}+b
$$

où $a$ et $b$ sont des coefficients scalaires constants, avec $a \geq 0, b \geq 0$

Au cours des deux saisons, le prix est déterminé par une fonction de demande inverse :

$$
p_{t, \mathrm{i}}^{\mathrm{c}}=\beta_{i}\left(q_{t, \mathrm{i}}^{\mathrm{c}}\right)^{-\alpha_{i}}
$$

où avec $\alpha_{i} \geq 0, \beta_{i}>0$. Il n'y a pas d'échanges en automne sur le marché local. Au cours de l'été, la production est vendue au commerçant par le producteur au prix de marché $p_{t, 2}^{l}$, lui-même une fonction linéaire du prix $p_{t, 2}^{\mathrm{c}}$ sur le marché central :

$$
p_{t, 2}^{l}=p_{t, 2}^{\mathrm{c}}-T
$$

où $T>0$ représente les coûts de transport et de manutention.

En automne, deux décisions doivent être prises. D'abord, les producteurs doivent décider de leur niveau de production $(h)$. Ensuite, le commerçant doit savoir ce qu'il vend tout de suite au consommateur final et ce qu'il stocke $(s)$. Tant le producteur que le commerçant ont de l'aversion pour le risque. Pour choisir la quantité $h_{t, 1}$ à produire, le producteur maximise $W_{f, t, 2}^{*}$, l'utilité qu'il espère pour l'été prochain (l'exposant* signifie ici qu'il $s^{\prime}$ agit d'anticipation). Comme le revenu espéré est $p_{t, 2}^{1 *} h_{t, 1}$, la valeur actuelle de l'équivalent certain $^{10}$ de ce revenu est donnée par :

$$
W_{f, t, 2}^{*} \frac{p_{t, 2}^{1 *} h_{t, 1}}{\overline{1}+r} \frac{A_{f} \operatorname{Var}^{*}\left(p_{t, 2}^{1 *} h_{t, 1}\right)}{1+r}
$$

10. On admet ici que l'équivalent certain est une combinaison linéaire de la moyenne et de la variance du gain espéré. On suit en cela le vieux modèle de Markowitz (1970). On pourrait évidemment faire usage d'un modèle plus moderne et plus satisfaisant que celui là. L'approche « moyenne/variance » présente cependant le mérite de la simplicité, tout en conservant une certaine pertinence dans les situations non extrêmes. En même temps, comme nous recherchons des « faits stylisés » plutôt que des estimations concrètes, ce niveau d'abstraction est probablement suffisant. 
où est le coefficient absolu d'aversion pour le risque du producteur ${ }^{11}, r$ le taux d'intérêt, et Var l'opérateur variance. À l'équilibre, le bénéfice marginal de la production $\frac{d W_{f, t, 2}^{*}}{d h_{t, 1}}$ est égal au coût marginal de production $p_{t, 1}$, donné par (1) et (3) ci-dessus. On a donc la fonction d'offre suivante :

$$
h_{t, 1}=\frac{\frac{p_{t, 2}^{l *}}{1+r}-b+T}{a+\frac{2 A_{f} \operatorname{Var}^{*}\left(p_{t, 2}^{l *}\right)}{1+r}}
$$

Les anticipations de prix sont « adaptatives », avec un décalage d'une année (deux saisons) pour tenir compte du caractère saisonnier de la production :

$$
p_{t, 2}^{l *}=p_{t-2,2}^{l *}-\lambda_{f}\left(p_{t-2,2}^{l *}-p_{t-2,2}^{l}\right)
$$

Bien entendu, $0 \leq \lambda_{f} \leq 1$. Finalement, $\operatorname{Var}^{*}\left(p_{t, 2}^{l *}\right)$ est estimé à partir d'un schéma d'anticipations naïves, comme chez Boussard (1996):

$$
\operatorname{Var}^{*}\left(p_{t, 2}^{l *}\right)=\left(p_{t-1,2}^{l}-p_{t-2,2}^{l}\right)^{2}
$$

De son côté, le commerçant décide le niveau de son stock en maximisant $W_{m, t, 2}^{*}$ l'utilité espérée de son revenu sur les deux saisons. $W_{m, t, 2}^{*}$ est donné par la valeur actuelle de l'équivalent certain du revenu, net de coût de stockage, moins le coût d'opportunité de la quantité stockée :

$$
W_{m, t, 2}^{*}=\frac{p_{t, 2}^{c^{*}}\left(s_{t-1,2}-s_{t, 1}\right)-A_{m} \operatorname{Var}^{*}\left(p_{t, 2}^{c^{*}}\left(s_{t-1,2}-s_{t, 1}\right)\right)}{1+r}-s_{t, 1} \delta-p_{t, 1}^{c} s_{t, 1}
$$

où $A_{m}$ est le coefficient d'aversion pour le risque du commerçant, et $\delta$ le coût de stockage. Bien sûr, il faut que : $0 \leq s_{t, 1} \leq s_{t-1,2}$.

Producteur et commerçant ont les mêmes types d'anticipations ${ }^{12}$. Pour obtenir l'utilité du commerçant, avec des anticipations « Nerloviennes », on peut donc dans (8) remplacer $p_{t, 2}^{c^{*}}$ $\operatorname{par} p_{t-2,2}^{c^{*}}-\lambda_{m}\left(p_{t-2,2}^{c^{*}}-p_{t-2,2}^{c}\right), \operatorname{Var}^{*}\left(p_{t, 2}^{c^{*}}\right) \operatorname{par}\left(p_{t-1,2}^{c}-p_{t-2,2}^{c}\right)^{2}$, et $p_{t, 1}^{c}$ par $\beta_{1}\left(s_{t-1,2}-s_{t-1}\right)^{-\alpha_{i}}$ donné par $(2)$

$W_{m, t, 2}^{*}=\frac{\left(p_{t-2,2}^{c^{*}}-\lambda_{m}\left(p_{t-2,2}^{c^{*}}-p_{t-2,2}^{c}\right)\right)\left(s_{t-1,2}-s_{t, 1}\right)-A_{m}\left(s_{t-1,2}-s_{t-1}\right)^{2}\left(p_{t-1,2}^{c}-p_{t-2,2}^{c}\right)^{2}}{1+r} s_{t, 1} \delta-\left(\beta_{1}\left(s_{t-1,2}-s_{t, 1}\right)^{-\alpha_{i}}\right) s_{t, 1}$

11. Il importe cependant de noter - comme le fait remarquer un des rapporteurs de cet article - que l'utilisation d'un coefficient d'aversion pour le risque constant est sans doute abusif, puisque celui-ci dépend de la richesse de l'agent économique représentatif (Bar-Shira, Just, Zilbernan, 1997), et que cette richesse varie au cours du cycle. Des travaux ultérieurs devront remédier à cette lacune.

12. Il semble raisonnable de supposer que les commerçants ont moins d'aversion pour le risque que les producteurs ( $c f$. Newbery, Stiglitz (1981), p. 164), ce que nous faisons lorsque nous prenons des valeurs du coefficient d'aversion pour le risque pour le commerçant et pour le producteur dans nos simulations. Toutefois, à notre connaissance, il n'y a pas de justification théorique ni empirique pour donner aux producteurs et aux commerçants des schémas d'anticipation différents. 
$s_{t, 1}$ est déterminé par la condition $\frac{d W_{m, t, 2}^{*}}{d s_{t, 1}}=0$. Une fois $s_{t, 1}$ fixé, les prix sur les deux marchés, central et local, respectivement sont donnés par $p_{t, 1}^{c}=\beta_{1}\left(s_{t-1,2}-s_{t, 1}\right)^{-\alpha_{i}}$ et par l'équation (3).

Pendant l'été, le commerçant détermine la quantité à garder en stock en maximisant :

$$
W_{m, t+1,1}^{*}=\frac{p_{t+1,1}^{c^{*}}\left(h_{t, 1}+s_{t, 1}-s_{t, 2}\right)-A_{m} \operatorname{Var}^{*}\left(p_{t+1,1}^{c^{*}}\left(h_{t, 1}+s_{t, 1}-s_{t, 2}\right)\right)}{1+r} \mathrm{~s}_{t, 2} \delta-p_{t, 2}^{c} s_{t, 2}
$$

sous la contrainte $0 \leq s_{t, 1} \leq C$, où $C$ est la capacité de stockage.

À nouveau, $s_{t, 2}$ est obtenu en résolvant $\frac{d W_{m, t+1,1}^{*}}{d s_{t, 2}}=0$. Les prix sont alors donnés par $p_{t, 2}^{c}=\beta_{2}\left(h_{t, 1}+s_{t, 1}-\mathrm{s}_{\mathrm{t}, 2}\right)^{-\alpha_{2}}$ et $p_{t, 2}^{l}=p_{t, 2}^{c}-T$

\section{Résultats du nouveau modèle et comparaison avec la théorie exogène}

Le modèle qui vient d'être présenté est un système dynamique complexe, qui ne peut faire l'objet d'une résolution analytique complète. Nous avons donc effectué une série de simulations numériques de ces équations en faisant varier les paramètres du modèle. Les intervalles des valeurs de paramètres ont été choisis en utilisant les valeurs trouvées dans la littérature empirique lorsque c'était possible ou des valeurs jugées plausibles.

Pour $\alpha_{i}$, une publication de Beghin, Bureau, et Drogué (2003) donne pour le marché coréen des valeurs d'élasticité directes Hicksiennes de la demande par rapport au prix allant de $-0,8$ pour le porc à 0,19 pour le riz. Cela correspond à des coefficients $\alpha_{i}$ variant de 5,3 à 1,25. Ici, nous avons fait des simulations et obtenons des résultats semblables lorsque $1<\alpha_{i}<2$.

Pour les élasticités d'offre, il est bien connu (et facile à vérifier) qu'avec une fonction de production de type Cobb Douglas à deux facteurs, l'un fixe, l'autre variable à prix constant, et telle que les exposants somment à un, si $s$ est la part du facteur variable dans la valeur de la production, alors l'élasticité de l'offre par rapport au prix est donnée par $s /(1-s)$ si les producteurs égalisent le coût marginal avec le prix. En supposant que la terre et le travail sont fixes, les consommations intermédiaires (estimées entre 20 et $40 \%$ de la valeur du produit) étant seules variables, on obtient une estimation de l'élasticité offre entre 16 et 0,66 . Il est clair que l'élasticité réelle se situe entre ces deux extrêmes. Ici, nous donnons des résultats pour une valeur de a proche de cette estimation $(a=0,5)$.

Pour les autres paramètres, nous avons choisi des valeurs jugées plausibles, ce qui n'est pas toujours évident : par exemple pour les coefficients d'aversion pour le risque, selon la théorie, ils devraient correspondre à l'agrégation (sous forme de moyenne harmonique) de coefficients d'aversion relative, multiplié par l'inverse de la « richesse » des sujets en cause. Il n'est pas facile de trouver des valeurs plausibles pour cela. Heureusement les propriétés qualitatives du modèle sont assez indépendantes de la valeur choisie dans un très vaste éventail (de 0,001 à 10 ). 

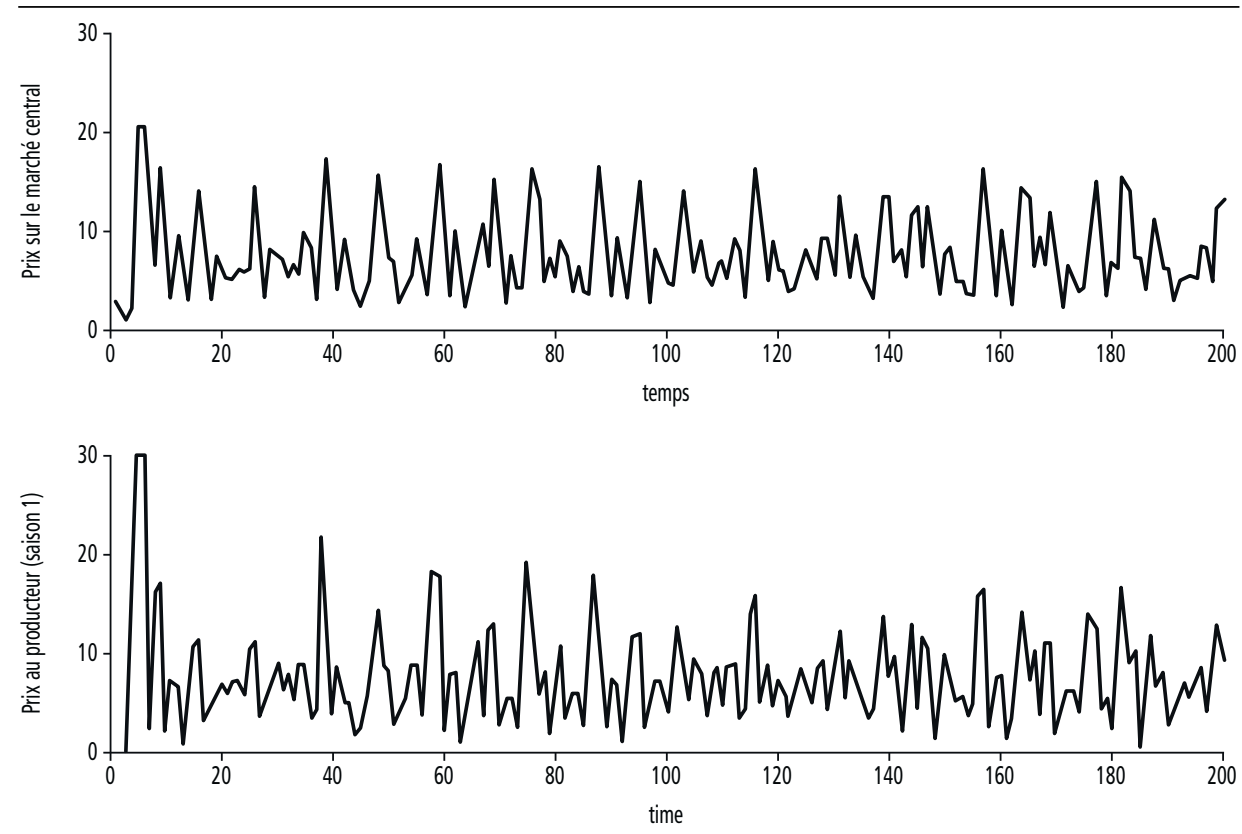

La figure 1 montre deux séries typiques obtenues de cette manière sur une période de 200 ans $^{13}$, l'une pour le marché local, l'autre pour le marché central. L'une et l'autre sont caractérisées par des mouvements autour de moyenne, avec de soudains pics très élevés. Ceux-ci surviennent quand les stocks sont épuisés. Il est intéressant de remarquer que les deux séries, quoique assez semblables, ne sont nullement affines. Les pics sur le marché central, survenant dans $19 \%$ des cas, sont plus élevés que ceux du marché local, et n'interviennent pas toujours au même moment. Il convient aussi de noter que ces résultats exhibent une auto-corrélation d'ordre 1 parfois positive, alors que les autres modèles chaotiques évoqués plus haut donnent toujours des valeurs négatives à cette caractéristique des séries de résultat ce qui était justement un motif pour les considérer comme peu réalistes. Comme c'était le cas dans le modèle de Deaton et

13. Dans toutes les simulations : $a_{i}=0,5 ; b_{i}=5$; $\alpha_{i}=0,6 ; \beta_{i}=1,3 ; T=1$.

Ici : $\lambda_{f}=0,1 ; \lambda_{m}=0,1 ; A_{f}=0,4 ; A_{m}=0,35 ; r=7 \%$; $\delta=0,1 ; T=1 ; a_{i}=0,5 ; b_{i}=5 ; \alpha_{i}=0,6 ; \beta_{i}=1,3$
Laroque mais dans un contexte tout différent - le stockage apparaît ainsi comme l'élément déterminant de ce trait majeur des séries de prix de matières premières.

Plus généralement, le modèle ci-dessus a été soumis à un test analogue à celui utilisé par Deaton et Laroque (1992). Nous avons fait varier systématiquement les paramètres du modèle, pour voir comment se déformait la série des résultats quand on modifie les valeurs des données qui semblent importantes pour les résultats. Le tableau 1 donne une idée des résultats obtenus, parmi des centaines. Il compare les caractéristiques de cinq séries simulées du marché central avec celles issues du même modèle sans stockage, celles de séries de prix annuels de matières premières ${ }^{14}$ recensées par Deaton et Laroque (op. cit.) et enfin les résultats de Deaton et Laroque

14. Les séries de prix couvrent la période 1900-1987 et les matières premières suivantes : bananes, cacao, café, cuivre, coton, jute, maïs, huile de palme, riz, sucre, thé, étain, et blé. Les données sur les prix sont issues de la Banque mondiale. Pour plus de détails sur ces données, voir Deaton et Laroque (op. cit.), p. 2. 
RECHERCHES

Sophie MITRA, Jean-Marc BOUSSARD

Tableau 1. Caractéristiques des séries observées et simulées

\begin{tabular}{|c|c|c|c|c|c|}
\hline & $\begin{array}{c}\text { Coefficient } \\
\text { de variation }\end{array}$ & $A C(1)$ & $A C(2)$ & Skewness & Kurtosis \\
\hline \multicolumn{6}{|c|}{ Simulations avec stockage } \\
\hline (a) & 0,51 & 0,44 & $-0,5$ & 0,79 & 3,39 \\
\hline (b) & 0,54 & $-0,33$ & 0,29 & 0,27 & 1,66 \\
\hline (c) & 0,51 & $-0,08$ & 0,41 & 0,94 & 3,22 \\
\hline (d) & 0,30 & 0,12 & 0,46 & 4,64 & 35,22 \\
\hline (e) & 0,48 & $-0,20$ & 0,30 & 0,80 & 3,11 \\
\hline \multicolumn{6}{|c|}{ Simulations sans stockage } \\
\hline (a) & 0,21 & $-0,19$ & 0,03 & $-0,63$ & 6,16 \\
\hline (b) & 0,24 & $-0,24$ & 0,03 & 0,08 & 5,17 \\
\hline (c) & 0,20 & 0,31 & $-0,23$ & 0,08 & 5,17 \\
\hline (d) & 0,20 & $-0,31$ & $-0,23$ & $-1,22$ & 6,65 \\
\hline (e) & 0,27 & $-0,22$ & 0,20 & 0,62 & 4,16 \\
\hline \multicolumn{6}{|c|}{ Séries réelles } \\
\hline Moyenne & 0,39 & 0,8 & 0,61 & 1,06 & 2,38 \\
\hline Minimum & 0,17 & 0,62 & 0,39 & 0,04 & $-0,98$ \\
\hline Maximum & 0,6 & 0,91 & 0,82 & 3,24 & 16,52 \\
\hline \multicolumn{6}{|c|}{ Deaton \& Laroque } \\
\hline Moyenne & 0,35 & 0,25 & 0,13 & 1,92 & 9,21 \\
\hline Minimum & 0,1 & 0,08 & 0,01 & 0,43 & $-0,29$ \\
\hline Maximum & 0,53 & 0,48 & 0,31 & 3,41 & 24,22 \\
\hline
\end{tabular}

Source : calculs des auteurs

(op. cit.). Le tableau donne le coefficient de variation, l'auto-corrélation de premier et de second rang, la Obliquité et la Kurtosis de séries simulées sur les principes ci-dessus, chacune correspondant à différentes valeurs des paramètres du modèle que sont l'élasticité d'anticipation, le taux d'intérêt, l'aversion pour le risque et le coût de stockage. Pour les 13 séries réelles, nous nous sommes limités à présenter la moyenne et les chiffres extrêmes.

Les paramètres qui changent sont :
(a) $\lambda_{f}=0,1 ; \lambda_{s}=0,1 ; A_{f}=0,4 ; A_{m}=0,35 ; r$ $=7 \% ; \delta=0,1$
(b) $\lambda_{f}=0,3 ; \lambda_{s}=0,3 ; A_{f}=0,4 ; A_{m}=0,35 ; r$ $=7 \% ; \delta=0,1$
(c) $\lambda_{f}=0,1 ; \lambda_{s}=0,1 ; A_{f}=0,4 ; A_{m}=0,35 ; r$ $=10 \% ; \delta=0,1$
(d) $\lambda_{f}=0,1 ; \lambda_{s}=0,1 ; A_{f}=0,25 ; A_{m}=0,10 ; r$ $=7 \% ; \delta=0,1$
(e) $\lambda_{f}=0,1 ; \lambda_{s}=0,1 ; A_{f}=0,4 ; A_{m}=0,35$; $r=7 \% ; \delta=0,7$

Avec des coefficients de variation de 30 à $54 \%$, les séries simulées tendent à être un peu plus volatiles que les séries réelles, sauf celles du café et du cacao pour lesquelles la volatilité est du même ordre. Le coefficient d'auto-corrélation d'ordre 1 est plus faible pour les séries simulées (il varie de $-0,08$ à $0,44)$ que pour les séries réelles, pour lesquelles il va de 0,62 à 0,91 . Il en est de même pour le coefficient du second ordre. Comme les séries réelles, les séries simulées ont une Obliquité positive, et donc une répartition dissymétrique. La Kurtosis des séries simulées varie de 1,66 à 35,22 , ce qui est en accord avec les « queues de distribution épaisses » que l'on observe pour les lois de probabilité des prix du sucre et d'autres matières premières agricoles. Il faut noter que nous obtenons des résultats semblables avec des séries simulées pour une période plus courte (100 ans au lieu de 200).

Au total, ces résultats sont très similaires à ceux obtenus par Deaton et Laroque avec un modèle de fluctuations exogènes. Ils ont aussi les mêmes défauts : par exemple, avec un coefficient maximum de 0,48 , Deaton et 
Laroque se plaignent de ne pouvoir reproduire les fortes auto-corrélations du premier ordre des séries réelles, au point d'avoir suscité les améliorations proposées par Cafiero et Wright (op. cit.), qui consistent à diminuer le coût de stockage, remontant par là le coefficient à 0,69 . Avec une autocorrélation de rang 1 maximale de 0,44, nos simulations donnent des résultats très voisins du modèle de Deaton et Laroque (op. cit.).

Plus intéressant est le fait que, en l'absence de stockage, notre modèle donne des séries qui convergent vers un équilibre, ou qui deviennent périodiques, obtenues avec le programme et les paramètres qui ont servis à construire la figure 1 , le seul changement étant la « capacité de stockage », maintenant imposée à zéro.

Ainsi se trouve mis en évidence le rôle du stockage privé dans ce contexte. Alors qu'avec des fluctuations quasi périodiques comme celles qui s'établissent ici, on pourrait penser, en s'appuyant sur la théorie « standard », qu'il suffirait de stocker pour faire disparaître les fluctuations, on voit sur la figure 1 que les fluctuations, si leur régime est modifié par rapport au cas sans stockage, ne sont nullement atténuées, bien au contraire. Il est intéressant de noter qu'en l'absence de stockage, c'est-à-dire en limitant la capacité de stockage à zéro, notre modèle a une dynamique tout à fait différente. Il donne alors des séries qui convergent vers un équilibre, ou qui deviennent périodiques, et exhibent ainsi moins de fluctuations comme le montre le coefficient de variation bien plus bas dans le cas sans stockage du tableau 1. Ce résultat suggère que, dans notre modèle, le stockage privé a un rôle déstabilisateur, puisqu'il est facteur de fluctuations endogènes des prix. C'est un résultat préliminaire qui a besoin d'être approfondi dans de futurs travaux. Il se situe à l'opposé du rôle stabilisateur qui a été attribué au stockage dans le contexte de l'hypothèse exogène (e.g., Newbery et Stiglitz, 1981 ; Williams et Wright, 1991) ${ }^{15}$. Ce résultat préliminaire est mathématiquement logique puisque le stockage, avec sa contrainte de non négativité, introduit une non-linéarité dans le modèle, condition nécessaire mais non suffisante pour obtenir une dynamique chaotique. D'un point de vue économique, on confirme ainsi le résultat théorique de MacKey (1989) qui trouve que le stockage spéculatif a un rôle déstabilisateur sur les prix, ou le résultat empirique de Fogel (1989) selon lequel en Angleterre, de 1500 à 1800 , les famines furent le résultat de l'inélasticité de la demande de stocks plus encore que des accidents météorologiques. Le stockage privé, longtemps supposé avoir un rôle stabilisateur sur les prix mérite d'être l'objet de davantage de travaux de recherche sur sa relation avec les fluctuations de prix.

Par souci de simplification, nous n'avons pas introduit les marchés à terme dans notre modèle ${ }^{16}$. Il est cependant clair que, dans la mesure où les marchés à terme constituent un système de stockage virtuel, ils devraient avoir les mêmes défauts et les mêmes qualités qu'un stockage réel, de sorte que les conclusions précédentes devraient s'y appliquer. Ceci serait de nature à conforter les analyses qui font jouer un rôle non négligeable à la spéculation dans l'explication de la crise récente (Mitchell, 2008) ${ }^{17}$.

15. En revanche, dans le contexte de stockage non compétitif, Chavas (2008) montre que le stockage contribue à faire fluctuer les prix.

16. À noter toutefois que Boussard (1996) a montré que l'introduction des marchés à terme ne change en rien la dynamique des séries chaotiques : simplement, l'aversion moyenne pour le risque du marché se trouve diminuée du fait que les «spéculateurs » ont moins d'aversion pour le risque que les producteurs, du moins en principe. Cela ne change pas le fonctionnement général du système.

17. Cf. Mitchell (2008), p. 15. 


\section{Conclusions}

Les fluctuations d'origine endogène peuvent donc reproduire les principales caractéristiques des séries réelles pour les prix des matières premières agricoles, comme le font les modèles basés sur des fluctuations exogènes, d'origine climatique ou autre. Il est clair, en même temps, qu'ils ne le font pas mieux que ces derniers, ce qui laisse encore de la place aux recherches sur l'origine de ces fluctuations. Les deux approches, endogène et exogène, de ces phénomènes restent donc encore difficiles à départager par la méthode expérimentale.

Pourtant, élucider ce point reste un problème crucial, puisque si le stockage compétitif privé conduit toujours à la même volatilité des prix, que les fluctuations soient d'origine exogène ou endogène, il faudrait développer d'autres méthodes pour réduire les fluctuations. Or ces méthodes, justement, diffèrent du tout au tout selon que l'origine des fluctuations est endogène ou exogène : la globalisation et la libéralisation devraient être efficaces si les fluctuations sont exogènes, elles seraient contre-productives avec des fluctuations endogènes.

Les réformes conduites depuis une vingtaine d'années dans de nombreux pays pour supprimer les institutions publiques et destinées à stabiliser les prix intérieurs ne semblent pas avoir réduit les fluctuations, bien au contraire : cela apporte de l'eau au moulin des théoriciens des fluctuations endogènes. Mais nous sommes encore bien loin d'avoir complètement compris le phénomène, et cela implique par conséquent un effort de recherche accru pour le faire. Le présent travail est une modeste contribution dans cette direction.

\section{RÉFÉRENCES BIBLIOGRAPHIQUES}

Alligood K., Sauer T.-D., Yorke J.-A. (1996). Chaos: An introduction to dynamical systems. Springer, New York. Bar-Shira, Z., Just, R.-E., and D. Zilbernan (1997). Estimation of Farmer's Risk Attitude: an Econometric Approach. Agricultural Economics, ${ }^{\circ} 17$, p. 211-222.

Bale M., Lutz E. (1979). The effect of Trade Intervention on International Price Instability, $A J A E$, vol.61, n 3, p. 512-516. Beghin J.-C. Bureau J.-C., Drogué S. (2003). The Calibration of Incomplete Demand Systems in Quantitative Analysis. Working paper, 2003/01 INRA Grigon.

Bobenrieth E., Bobenrieth J., Wright B.-D. (2006). Theoretical Advances in the Modeling of Storage and Speculation. In Sarris A., Hallam D. (eds.) "Agricultural Markets and Trade FAO », Rome, p. 115-133.

Boussard J.-M. (1996). When Risk Generates Chaos. Journal of Economic Behavior and Organization, $\mathrm{n}^{\circ} 29$, p. 433-46.
Boussard J.-M. (2006). Price Risk Management Instruments in Agricultural and Other Unstable Markets. The ICFAI Journal of Risk and Insurance, $\mathrm{n}^{\circ} 3$, p. 6-19.

Brock W.-A., Dindo P.-D.-E., Hommes C.-H. (2007). Adaptive rational equilibrium with forward looking agents. International Journal of Economic Theory, $\mathrm{n}^{\circ} 2$, p. 241-278.

Cafiero C., Wright B.-D. (2006). Is the storage model a 'closed' empirical issue? The empirical ability of the storage model to explain price dynamics. In Sarris A., Hallam D. (eds.), Agricultural Markets and Trade FAO, Rome, p. 89-115.

Chavas J.-P. (2008). On Storage Behaviour under Imperfect Competition, with application to the American Cheese Market. Review of Industrial Organisation, $\mathrm{n}^{\circ} 33$, p. 325-339

Chavas J.-P., Holt M.-T. (1993). Market Instability and Nonlinear Dynamics, 
American Journal of Agricultural Economics, $\mathrm{n}^{\circ}$ 75, p. 113-20.

Cobble K.-H., Dismuke R., Thomas S. (2007). Policy implication of crop yields and revenue variability at differing levels of disaggregation. Portland, Paper presented at the annual meeting of the American association of agricultural economy.

Deaton A., Laroque G. (1992). On the Behavior of Commodity Prices. Review of Economic Studies, n ${ }^{\circ}$ 59, p. 1-23.

Deaton A., Laroque G. (1996). Competitive Storage and Commodity Price Dynamics. Journal of Political Economy, $\mathrm{n}^{\circ} 104, \mathrm{p}$. 896-923.

Ezekiel M. (1938). The Cobweb Theorem. Quarterly Journal of Economics, $\mathrm{n}^{\circ} 53$, p. 225-280.

Finkenstadt B., Kuhbier P. (1992). Chaotic Dynamics in Agricultural Markets. Annals of Operations Research, $\mathrm{n}^{\circ} 37$, p. 73-96.

Fogel R.-W. (1989). Second Thoughts on the European Escape from Hunger: Famines, Price Elasticities, Entitlements, Chronic Malnutrition, and Mortality Rates. Working Paper, $\mathrm{n}^{\circ} 1$, National Bureau of Economic Research Working Paper Series on Historical Factors in Long-Run Growth.

Hommes C.-H. (1998). On the Consistency of Backward-Looking Expectations: The Case of the Cobweb. Journal of Economic Behavior and Organization, $\mathrm{n}^{\circ} 33$, p. 333-62.

Hommes C.-H., van Eekelen A. (1996). Partial Equilibrium Analysis in a Noisy Chaotic Market. Economics Letters, $\mathrm{n}^{\circ} 53$, p. 275-282.
Leuthold R.-M., Wei A. (1998). Long Agricultural Futures Prices: ARCH, Long Memory or Chaos Processes? Mimeo, OFOR papers, $\mathrm{n}^{\circ}$ 98-3, May.

MacKey M.-C. (1989). Commodity Price Fluctuations: Price Dependent Delays and Nonlinearities as Explanatory Factors. Journal of Economic Theory, vol. 48, p. 497-509.

Mandelbrot B. (1973). Le problème de la réalité des cycles lents et le syndrome de Joseph. Economie appliqué, vol. 26, n 2 , p. 349-364.

Markowitz H.-M. (1970). Portfolio analysis: Efficient diversification of investments. Yale, Yale university press.

Mitchell D. (2008). A Note on Rising Food Prices. Policy Research Working Paper, The World Bank.

Nerlove M., Bessler D.-A. (2001) Expectation, Information and Dynamics, Handbook of Agricultural Economics, vol. 1A.

Newbery D.-M.-G., Stiglitz J.-E. (1981). The Theory of Commodity Price Stabilization. Oxford, Clarendon press.

Orléans A. (1989). Comportements mimétiques et diversité des opinions sur les marchés financiers. In Bourguinat H., Artus P. (dirs.), «Théorie économique et crises des marchés financiers », Paris, Economica.

Ravallion M. (1997). Famines and Economics. Journal of Economic Literature, $\mathrm{n}^{\circ} 35$, p. 1205-1242.

Williams J.-C., Wright B.-D. (1991). Storage and Commodity Markets. Cambridge, U.K, Cambridge University Press.

Zajdenweber D. (2000). Économie des extrêmes. Paris, Economica. 